\title{
FIBROMA TIPO NUCAL LATEROCERVICAL. DESCRIPCIÓN DE UN CASO
}

\section{Laterocervical nuchal-type fibroma. A case report}

Fernando GARCÍA-CURDI ${ }^{1}$; Pamela Ellyette BENÍTEZ-ALONSO²; Yolanda LOIS-ORTEGA ${ }^{1}$; Manuel MOROSGARCÍA ${ }^{3}$; José Miguel SEBASTIÁN-CORTÉS ${ }^{1}$; Héctor VALLÉS-VARELA ${ }^{1}$

${ }^{1}$ Hospital Clínico Universitario Lozano Blesa. Servicio de Otorrinolaringología. Zaragoza. España. ${ }^{2}$ Complejo Hospitalario de Navarra. Servicio de Otorrinolaringología. Pamplona. España. ${ }^{3}$ Hospital Clínico Universitario Lozano Blesa. Servicio de Anatomía Patológica. Zaragoza. España.

Correspondencia:fgcurdi@gmail.com

Fecha de recepción: 18 de mayo de 2018

Fecha de aceptación: 13 de junio de 2018

Fecha de publicación: 17 de junio de 2018

Fecha de publicación del fascículo: 1 de diciembre de 2019

Conflicto de intereses: Los autores declaran no tener conflictos de intereses

Imágenes: Los autores declaran haber obtenido las imágenes con el permiso de los pacientes

Política de derechos y autoarchivo: se permite el autoarchivo de la versión post-print (SHERPA/RoMEO)

Licencia CC BY-NC-ND. Licencia Creative Commons Atribución-NoComercial-SinDerivar 4.0 Internacional

Universidad de Salamanca. Su comercialización está sujeta al permiso del editor

RESUMEN: Introducción: El Fibroma tipo nucal es una rara proliferación de tejidos blandos, de carácter benigno. Aparece de manera más frecuente en hombres de edad media, habitualmente como una masa solitaria y menor de $6 \mathrm{~cm}$, pobremente circunscrita, no encapsulada, de consistencia densa y de coloración blanquecina. Su etiopatogenia es desconocida y habitualmente es asintomático. Su diagnóstico es histológico, y su tratamiento la resección completa. Descripción del caso: Presentamos el caso de una paciente de 72 años que acude a urgencias por hallazgo casual de tumoración laterocervical derecha hace 5 días, no refiere disfonía, tampoco odinofagia, otalgia, disnea, fiebre o síndrome constitucional. Tras la exploración y las pruebas de imagen necesarias, se interviene quirúrgicamente con buenos resultado, resultado tratarse de un fibroma tipo nucal de localización laterocervical. Conclusiones: La evaluación clínica de una masa solitaria del cuello puede ser difícil por lo que el fibroma tipo nucal debe incluirse entre los posibles diagnósticos diferenciales de tumoraciones en la región cervical posterior.

Palabras clave: fibroma tipo nucal; tumor cervical. 


\begin{abstract}
SUMMARY: Introduction: Nuchal-type fibroma is a rare proliferation of soft tissues of a benign nature. It appears more frequently in middle-aged men, usually as a solitary mass and less than $6 \mathrm{~cm}$, poorly circumscribed, not encapsulated, dense and whitish in color. Its etiopathogenesis is unknown and is usually asymptomatic. Its diagnosis is histological, and its treatment the complete resection. Case description: We present the case of a 72-year-old patient who came to our hospital due to the chance finding of a right laterocervical tumor 5 days ago. She does not treat dysphonia, odynophagia, otalgia, dyspnea, fever or constitutional syndrome. After the exploration and the necessary imaging tests, it is surgically intervened with good results, the result is a type of fibroma of laterocervical localization. Conclusions: The clinical evaluation of a solitary neck mass can be difficult, so the nuchal fibroid should include the possible differential diagnoses of tumors in the posterior cervical region.
\end{abstract}

Keywords: nuchal-type fibroma; cervical mass.

\section{INTRODUCCIÓN}

El fibroma tipo nucal fue descrito en 1988 por Enzinger y Weiss, es una tumoración benigna de etiología desconocida, formada por una proliferación del tejido fibroso dérmico y subcutáneo [2, 3]. Su crecimiento es lento, de años de evolución, oscilando el diámetro entre 2.5 y $8 \mathrm{~cm}$ [4], habiéndose publicado un caso con un tamaño de $20 \mathrm{~cm}$ [2]. Es típica su aparición en pacientes varones de mediana edad, clínicamente se presenta como una masa solitaria en la región posterior del cuello [5]. Sin embargo, puede tener una localización extranucal, cuando esto ocurre, los lugares donde más frecuentemente se asientan son la espalda (región interescapular), el hombro y la cara. Su diagnóstico se basa en el estudio histológico, donde se observan haces de colágeno entremezclados con tejido adiposo y nervios periféricos, con escasas fibras de elastina [2], y ocasionalmente, músculo esquelético $[4,6]$. Las células son muy positivas para vimentina, CD34 y algunas veces CD99. Hasta en $2 / 3$ de los casos puede haber una reacción nuclear con B-catenina [7]. La prueba de imagen de elección es la resonancia magnética (RNM), debido a la gran precisión con que muestra los tejidos blandos. Mediante esta prueba se observa (tanto en T1 como en T2) como una masa con baja intensidad de sin realce significativo debido a su red fibrosa densa y contenido mínimo de agua [4]. El tratamiento definitivo es la exéresis quirúrgica, con un seguimiento posterior para descartar recidiva del proceso.

\section{DESCRIPCIÓN}

Presentamos el caso de una paciente de 72 años que acudió a urgencias por hallazgo casual de una tumoración laterocervical derecha de 5 días de evolución, no refería disfonía, ni odinofagia, otalgia, disnea, fiebre o síndrome constitucional. La paciente no tenía antecedentes personales de interés, ni hábitos tóxicos. En la exploración física se palpaba una tumoración laterocervical derecha en el área IV, de unos 3-4 cm de diámetro, adherida a planos profundos, no pulsátil y no dolorosa a la palpación. Se realizó una rinofibrolaringoscopia que no mostró hallazgos patológicos. La impresión diagnóstica fue de adenopatía laterocervical derecha. Se solicitó una punción-aspiración con aguja fina (PAAF), como estudio complementario, la cual no fue concluyente; en la tomografía computarizada (TC), se visualizaba una masa ovalada de unos $22 \times 16 \mathrm{~mm}$, localizada por detrás del tercio inferior del músculo esternocleidomastoideo que impresionaba de conglomerado adenopático (Figura 1), y en la Tomografía por Emisión de Positrones-TC (PET-TC ${ }^{18--F D G}$ ) adenopatías laterocervicales derechas ligeramente hipermetabólicas y una pequeña adenopatía hipermetabólica menor de $1 \mathrm{~cm}$ con un índice SUVMAX de 3 (Figura 1). Ante la exploración y el estudio de imagen se propuso intervención quirúrgica que la paciente aceptó. Durante la intervención se apreció una tumoración dura, blanca, fija a planos profundos de $4 \mathrm{~cm}$ de diámetro, que infiltraba el músculo esternocleidomastoideo derecho en su cara interna, y a la vena yugular interna. El análisis anatomopatológico 
reveló un tumor mesenquimal fusocelular que englobaba tejido adiposo, infiltraba músculo esquelético y a la pared de la yugular proyectándose en su luz, que presentaba frecuentes fibras elásticas y que se extendía a margen quirúrgico cauterizado con el bisturí eléctrico; lo que era compatible con fibroma de tipo nucal (Figura 2). Actualmente la paciente se encuentra asintomática, con una buena cicatrización de la herida quirúrgica y sin ninguna complicación postoperatoria.

\section{DISCUSIÓN}

Los fibromas tipo nucal son tumores raros, benignos y fibrosos que se presentan como masas subcutáneas asintomáticas, duras y poco circunscritas que se desarrollan típicamente en la región posterior del cuello, pero aproximadamente en un tercio de los casos aparecen en otras localizaciones $[1,2,3]$ que son morfológica e histológicamente indistinguibles de las de la región nucal; por lo que, en 1999 Michal et al. propusieron el término «fibroma de tipo nucal» para agrupar aquellas lesiones histológicamente similares al fibroma nucal, independientemente de su sitio de origen $[8,9]$.

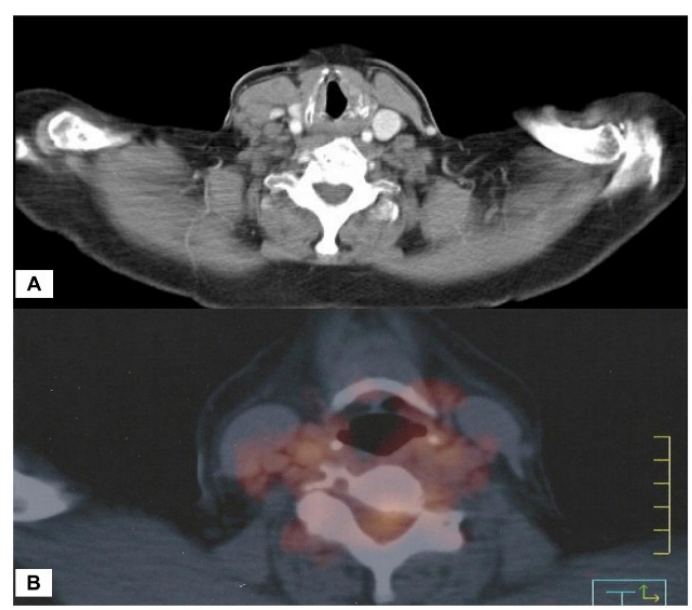

Figura 1. Pruebas de imagen. A) TC: corte axial en el que se aprecia una masa cervical derecha por detrás del músculo esternocleidomastoideo. B) PET-TC ${ }^{18 F-\text { FDG}}$; corte axial en el que se identifican adenopatías laterocervicales derechas ligeramente hipermetabólicas sin identificar neoplasia primaria.

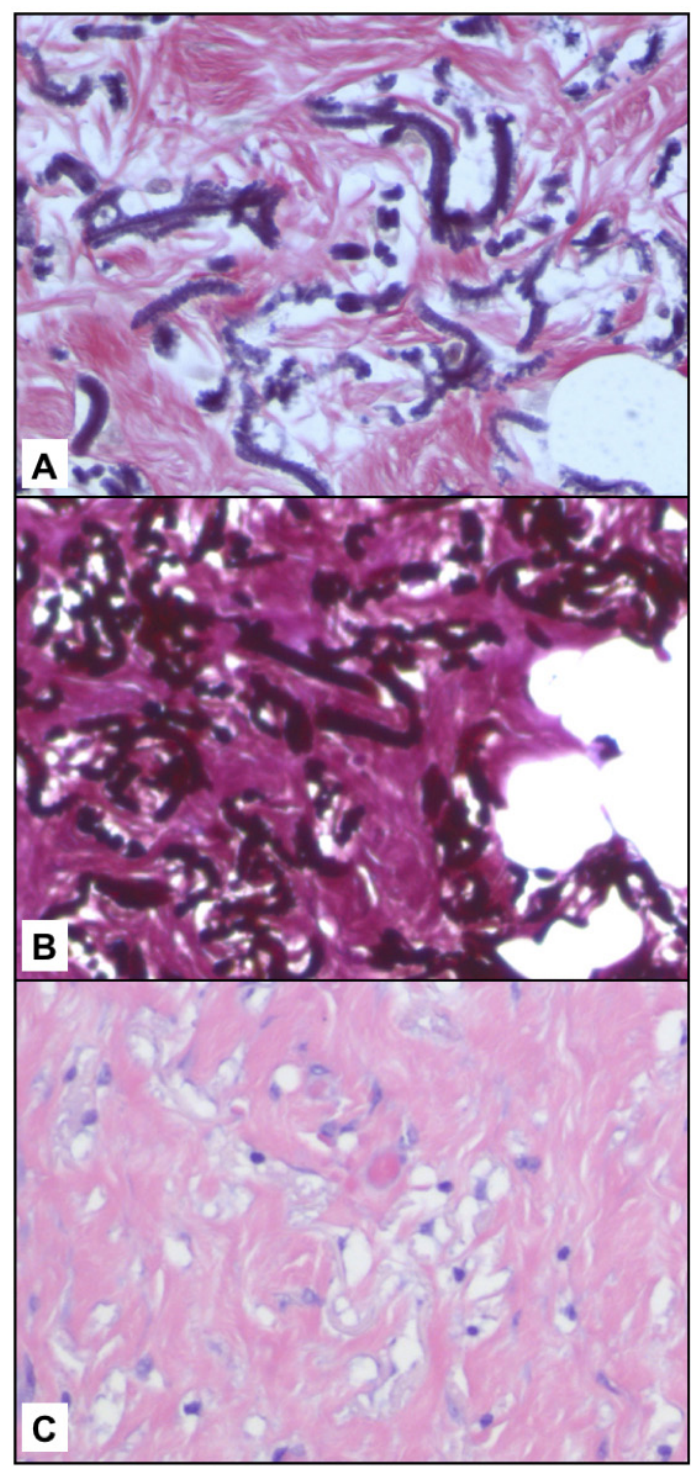

Figura 2. Estudio histológico de la pieza. A) Tinción de Van Gieson. Se puede observar en color rosa las fibras de colágeno, y en morado las fibras elásticas, que en su sección transversal tienen froma de disco. B) Tinción de Orceína. Se identifica un entramado de fibras de colágeno. Dentro en color marrón fibras elásticas y en blanco; tejido adiposo. C) Tinción de Hematoxilina - Eosina. Proliferación fibroblástica fusocelular y de colágeno. Se aprecian fibras elásticas embebidas en la matriz conectiva. 


\section{FIBROMA TIPO NUCAL LATEROCERVICAL. DESCRIPCIÓN DE UN CASO GARCÍA-CURDI F ET AL}

Su patogenia no está descrita todavía, pero algunos casos se han asociado con traumatismos, diabetes mellitus y síndrome de Gardner. Sin embargo, se han descrito los hallazgos que encontraremos en una visión microscópica de la pieza: fibras de colágeno mezclado con tejido adiposo y nervios periféricos, con escasa elastina [6].

Muchos casos de fibroma tipo nucal han sido diagnosticados erróneamente debido a su evolución clínica asintomática y su similitud histopatológica con otros tumores fibrosos benignos. Debe hacerse un exhaustivo diagnóstico diferencial con otras entidades como la fibromatosis de tipo desmoide, colagenoma estoriforme circunscrito, fibrolipoma, elastofibroma y tejido cicatricial $[7,8]$.

Existen pocos casos descritos en la literatura con esta patología. Debemos sospechar que pueda tratarse de un fibroma tipo nucal ante una masa asintomática de un tamaño aproximado de $3 \mathrm{~cm}$ en la región posterior del cuello [9]. El tratamiento de elección es la resección quirúrgica completa, sin embargo, el hecho de que sea un tumor no encapsulado dificulta la escisión completa, y puede contribuir a la aparición de recidivas locales, lo que obliga a un seguimiento posterior del paciente $[10,11]$.

\section{CONCLUSIONES}

En el caso descrito las características macroscópicas e histológicas de la tumoración se asemejaban a los casos descritos en la literatura. Tras practicar su resección completa, la paciente permanece asintomática y sin signos de recidiva. Debido a la extraordinaria localización del tumor, así como de su estirpe anatomopatológica, consideramos importante presentar este caso.

La evaluación clínica de una masa solitaria del cuello puede ser difícil por lo que el fibroma tipo nucal debe incluirse entre los posibles diagnósticos diferenciales de tumoraciones en la región cervical posterior, siendo el estudio histológico determinante para su confirmación.

\section{BIBLIOGRAFÍA}

1. Enzinger FM, Weiss SW. Benign tumors and tumorlike lesions of fibrous tissue. In: Enzinger FM, Weiss SW, editors. Soft tissue tumors. 2nd ed. St. Louis: Mosby; 1988. pp. 102-35.

2. Gong Y, Zhao X, Wu DI, Liu J. Nuchal-type fibroma of the shoulder: A case report and review of the literature. Oncol Lett. 2016; 11:4152-4.

3. Alsaleh $\mathrm{N}$, Amanguno $\mathrm{H}$. Nuchal Fibroma: A rare entity of neck masses. Gulf J Oncolog. 2015;1:10-2.

4. Lee GK, Suh KJ, Lee SM, Lee SJ. Nuchal-type fibroma of the buttock: magnetic resonance imaging findings. Jpn J Radiol. 2010;28:538-41.

5. Hameed M, Benevenia J, Blacksin M, Aisner SC. Nuchal fibroma of the shoulder involving skeletal muscle: a radio- graphic and clinicopathological study-a case report. J Bone Joint Surg Am 1998;80:1684-6.

6. Lee SE, Kim YC, Kim SC. Nuchal fibroma presenting as two posterior neck masses. J Dermatol. 2007;34:262-3.

7. Michal M, Fetsch JF, Hes O, and Miettinen M. "Nuchal- type fibroma: a clinicopathologic study of 52 cases". Cancer. 1999;85:156-63.

8. Kim DH, Kim TH, Sung NH, Shin H, Lee AY, Lee SH. Multiple nuchal-type fibromas on the scalp: a case report. Ann Dermatol. 2015;27:194-6.

9. Michal M, Fetsch JF, Hes O, Miettinen M. Nuchaltype fibroma: A clinicopathologic study of 52 cases. Cancer. 1999;85:156-63.

10. Shin JB, Son SW, Kim IH. Nuchal-type Fibroma of the Coccyx. Ann Dermatol.2008;20:41-4.

11. Sraj SA, Lahoud LE, Musharafieh R, Taha A. Nuchal-type fibroma of the ankle: a case report. J Foot Ankle Surg. 2008;47:332-6. 